\title{
Preliminary Estimate of Excess Mortality During the COVID-19 Outbreak - New York City, March 11-May 2, 2020
}

\author{
New York City Department of Health and Mental Hygiene (DOHMH) COVID-19 Response Team
}

\begin{abstract}
On May 11, 2020, this report was posted as an MMWR Early Release on the MMWR website (https://www.cdc.gov/mmwr).
\end{abstract}

SARS-CoV-2, the virus that causes coronavirus disease 2019 (COVID-19), was first identified in December 2019 in Wuhan, China, and has since spread worldwide. On March 11, 2020, the World Health Organization declared COVID-19 a pandemic (1). That same day, the first confirmed COVID-19associated fatality occurred in New York City (NYC). To identify confirmed COVID-19-associated deaths, defined as those occurring in persons with laboratory-confirmed SARS-CoV-2 infection, on March 13, 2020, the New York City Department of Health and Mental Hygiene (DOHMH) initiated a daily match between all deaths reported to the DOHMH electronic vital registry system (eVital) (2) and laboratory-confirmed cases of COVID-19. Deaths for which COVID-19, SARS-CoV-2, or an equivalent term is listed on the death certificate as an immediate, underlying, or contributing cause of death, but that do not have laboratory-confirmation of COVID-19 are classified as probable COVID-19-associated deaths. As of May 2, a total of 13,831 laboratory-confirmed COVID-19-associated deaths, and 5,048 probable COVID-19-associated deaths were recorded in NYC (3). Counting only confirmed or probable COVID-19-associated deaths, however, likely underestimates the number of deaths attributable to the pandemic. The counting of confirmed and probable COVID-19-associated deaths might not include deaths among persons with SARS-CoV-2 infection who did not access diagnostic testing, tested falsely negative, or became infected after testing negative, died outside of a health care setting, or for whom COVID-19 was not suspected by a health care provider as a cause of death. The counting of confirmed and probable COVID-19-associated deaths also does not include deaths that are not directly associated with SARS-CoV-2 infection. The objective of this report is to provide an estimate of all-cause excess deaths that have occurred in NYC in the setting of widespread community transmission of SARS-CoV-2. Excess deaths refer to the number of deaths above expected seasonal baseline levels, regardless of the reported cause of death. Estimation of all-cause excess deaths is used as a nonspecific measure of the severity or impact of pandemics (4) and public health emergencies (5). Reporting of excess deaths might provide a more accurate measure of the impact of the pandemic.
DOHMH has developed an electronic vital statistics reporting system that provides a near complete count of all deaths that occur in NYC (6). Rapid reporting of the event of death using this electronic system allows timely surveillance of all deaths in NYC (i.e., all-cause mortality) pending complete recording of demographic and International Classification of Diseases, Tenth Revision (ICD-10) coding of cause of death information. To estimate excess deaths in NYC during the COVID-19 pandemic, a seasonal periodic regression model, as is routinely conducted for monitoring the impact of seasonal influenza (7), was used. Excess deaths were determined for the period March 11-May 2, 2020, using mortality data from the period January 1, 2015-May 2, 2020 and calculated as the difference between the seasonally expected baseline number and the reported number of all-cause deaths $(7,8)$. A limitation of this approach is that it does not account for uncertainty in the reporting lag or completeness of these provisional data.

During March 11-May 2, 2020, a total of 32,107 deaths were reported to DOHMH; of these deaths, 24,172 (95\% confidence interval $=22,980-25,364$ ) were found to be in excess of the seasonal expected baseline. Included in the 24,172 deaths were 13,831 (57\%) laboratory-confirmed COVID-19-associated deaths and 5,048 (21\%) probable COVID-19-associated deaths, leaving 5,293 (22\%) excess deaths that were not identified as either laboratory-confirmed or probable COVID-19-associated deaths (Figure).

The 5,293 excess deaths not identified as confirmed or probable COVID-19-associated deaths might have been directly or indirectly attributable to the pandemic. The percentages of these excess deaths that occurred in persons infected with SARS-CoV-2 or resulted from indirect impacts of the pandemic are unknown and require further investigation.

COVID-19-associated mortality is higher in persons with underlying chronic health conditions such as heart disease and diabetes (9), and deaths in persons with these chronic health conditions might not be recognized as being directly attributable to COVID-19. In addition, social distancing practices, the demand on hospitals and health care providers, and public fear related to COVID-19 might lead to delays in seeking or obtaining lifesaving care. Thus, monitoring of all-cause deaths and estimating excess mortality during the pandemic provides a more sensitive measure of the total number of deaths than 
FIGURE. Number of laboratory-confirmed* and probable ${ }^{\dagger}$ COVID-19-associated deaths and total estimated excess deaths ${ }^{\S}$ - New York City, March 11-May 2, 2020

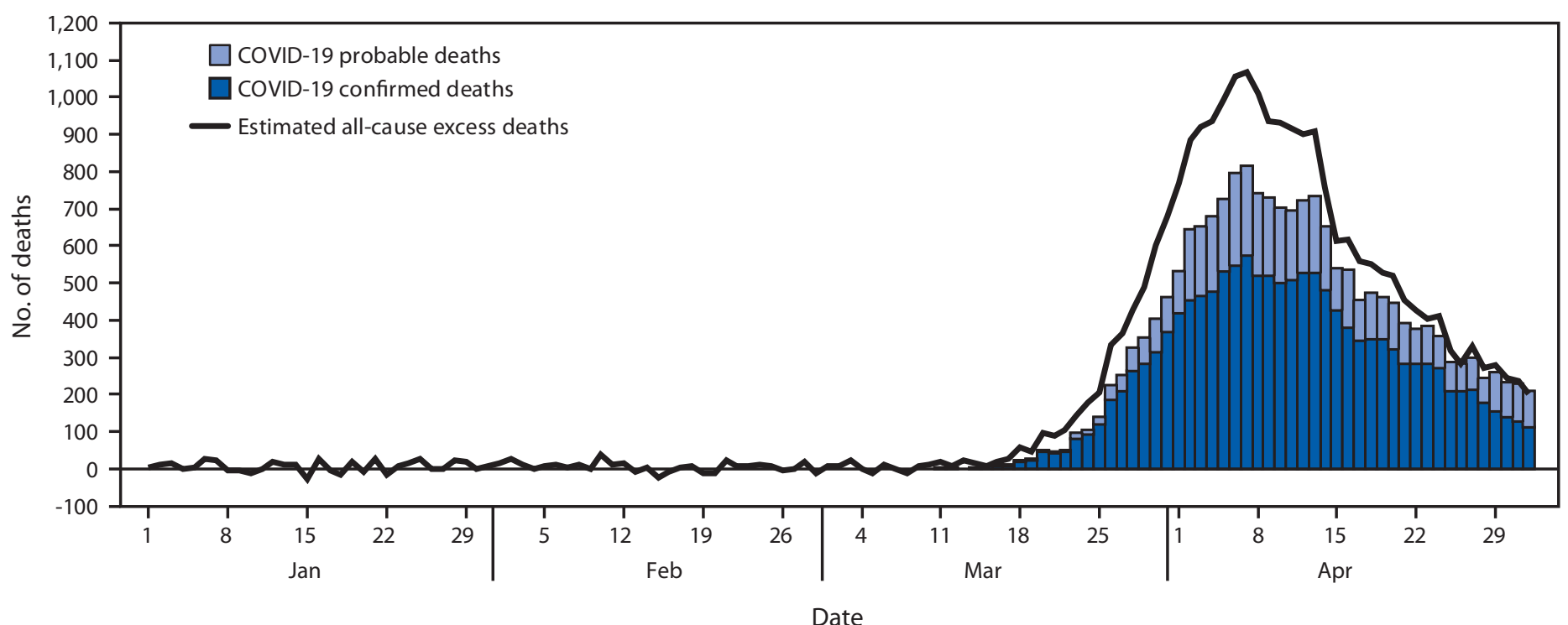

* Death in a person with a positive laboratory test for SARS-CoV-2 RNA.

† Death in a person without a positive test for SARS-CoV-2 RNA but for whom COVID-19, SARS-CoV-2, or a related term was listed as an immediate, underlying, or contributing cause of death on the death certificate.

$\S$ Total excess all-cause deaths were calculated as observed deaths minus expected deaths as determined by a seasonal regression model using mortality data from the period January 1, 2015-May 2, 2020.

would be recorded by counting laboratory-confirmed or probable COVID-19-associated deaths.

This approach can account for factors temporally, but not causally, associated with SARS-CoV-2 that might affect death rates, including other pathogens circulating during the overlapping 2019-20 influenza season. All-cause mortality surveillance based on electronic reporting of the event of death provides a faster and more inclusive measure of the pandemic's impact on mortality than does relying only on national COVID-19 reporting mechanisms (10).

\section{Acknowledgments}

Bureau of Vital Statistics team; staff members in the NYC DOHMH Incident Command System Surveillance and Epidemiology Section.

\section{New York City Department of Health and Mental Hygiene (DOHMH) COVID-19 Response Team}

Donald R. Olson, DOHMH; Mary Huynh, DOHMH; Annie Fine, DOHMH; Jennifer Baumgartner, DOHMH; Alejandro Castro, DOHMH; Hiu Tai Chan, DOHMH; Demetre Daskalakis, DOHMH; Katelynn Devinney, DOHMH; Kevin Guerra, DOHMH; Scott Harper, DOHMH, CDC; Joseph Kennedy, DOHMH; Kevin Konty, DOHMH; Wenhui Li, DOHMH; Emily McGibbon, DOHMH; Jaimie Shaff, DOHMH; Corinne Thompson, DOHMH; Neil M. Vora, DOHMH, CDC; Gretchen Van Wye, DOHMH.

Corresponding authors: Donald R. Olson, dolson@health.nyc.gov; Gretchen Van Wye, gvanwye@health.nyc.gov.

All authors have completed and submitted the International Committee of Medical Journal Editors form for disclosure of potential conflicts of interest. No potential conflicts of interest were disclosed. 


\section{References}

1. World Health Organization. WHO Director-General's opening remarks at the media briefing on COVID-19, March 2020. Geneva, Switzerland: World Health Organization; 2020. https://www.who.int/dg/speeches/ detail/who-director-general-s-opening-remarks-at-the-media-briefingon-covid-19---11-march-2020

2. New York City Department of Health and Mental Hygiene. eVital. New York NY: City of New York; 2020. https://www1.nyc.gov/site/doh/ providers/reporting-and-services/evital.page

3. New York City Department of Health and Mental Hygiene. Covid-19: data. New York NY: City of New York; 2020. https://www1.nyc.gov/ site/doh/covid/covid-19-data.page

4. Simonsen L, Clarke MJ, Williamson GD, Stroup DF, Arden NH, Schonberger LB. The impact of influenza epidemics on mortality: introducing a severity index. Am J Public Health 1997;87:1944-50. https://doi.org/10.2105/AJPH.87.12.1944

5. Santos-Burgoa C, Sandberg J, Suárez E, et al. Differential and persistent risk of excess mortality from Hurricane Maria in Puerto Rico: a timeseries analysis. Lancet Planet Health 2018;2:e478-88. https://doi. org/10.1016/S2542-5196(18)30209-2
6. Castro A, Li W, Huynh M, Van Wye G. Development of the mortality syndromic surveillance system (MortalSS)—New York City. In: American Public Health Association Annual Meeting \& Expo, October 29-November 2; Denver, CO.

7. CDC. Fluview. U.S. Influenza Surveillance System: purpose and methods. Atlanta, GA: US Department of Health and Human Services, CDC; 2020. https://www.cdc.gov/flu/weekly/overview.htm

8. Pelat C, Boëlle PY, Cowling BJ, et al. Online detection and quantification of epidemics. BMC Med Inform Decis Mak 2007;7:29. https://doi. org/10.1186/1472-6947-7-29

9. Chow N, Fleming-Dutra K, Gierke R, et al.; CDC COVID-19 Response Team. COVID-19 Response Team. Preliminary estimates of the prevalence of selected underlying health conditions among patients with coronavirus disease 2019—United States, February 12-March 28, 2020. MMWR Morb Mortal Wkly Rep 2020;69:382-6. https://doi. org/10.15585/mmwr.mm6913e2

10. Bialek S, Bowen V, Chow N, et al.; CDC COVID-19 Response Team. COVID-19 Response Team. Geographic differences in COVID-19 cases, deaths, and incidence-United States, February 12-April 7, 2020. MMWR Morb Mortal Wkly Rep 2020;69:465-71. https://doi. org/10.15585/mmwr.mm6915e4 